\title{
WSN Based Temperature Monitoring System for Multiple Locations in Industry
}

\author{
Aditya Jogdand, Amit Chaudhari, Niranjan Kadu, Udaykumar Shroff \\ Department of Computer Engineering, MET's Institute of Engineering, Nashik, Maharashtra, India
}

\begin{abstract}
How to cite this paper: Aditya Jogdand | Amit Chaudhari | Niranjan Kadu | Udaykumar Shroff "WSN Based Temperature Monitoring System for Multiple Locations in Industry" Published in International Journal of Trend in Scientific Research and Development (ijtsrd), ISSN: 24566470, Volume-3 | Issue-4, June 2019, pp.8-11, URL: https://www.ijtsrd.c om/papers/ijtsrd23 124.pdf

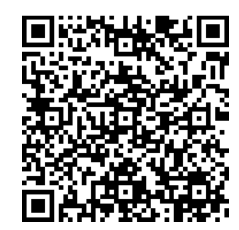
IITSRD23124

Copyright (C) 2019 by author(s) and International Journal of Trend in Scientific Research and Development Journal. This is an Open Access article distributed under the terms of the Creative Commons

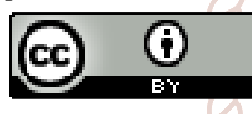
Attribution License (CC B 4.0) (http://creativecommons.org/licenses/ by/4.0)
\end{abstract}

\section{Introduction}

In this world we are facing number of emergency problems so that to response to such type of emergencies we need to design a wireless network which responds to such emergency and possible to control it. Temperature monitoring is possible using wireless network also controlling of temperature is also possible.If we are able to design a such type of network then definitely in future we can avoid property from damage and also we can save life of people

\subsection{Wireless Sensor Networks}

Wireless sensor network (WSN) refers to a group of spatially dispersed and dedicated sensors for monitoring and recording the physical conditions of the environment and organizing the collected data at a central location. WSNs measure environmental conditions like temperature, sound, pollution levels, humidity, wind, and so on.The WSN is built of "nodes" from a few to several hundreds or even thousands, where each node is connected to one (or sometimes several) sensors. Each such sensor network node has typically several parts: a radio transceiver with an internal antenna or connection to an external antenna, a microcontroller, an electronic circuit for interfacing with the sensors and an energy source, usually a battery or an embedded form of energy harvesting.Here We are using
DHT11 for Sensing temperature, Arduino Nano Microprocessor and NRF Transciever as a radio transceiver.

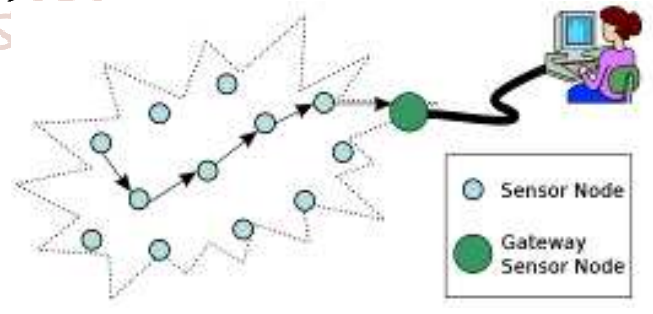

\section{Literature Survey}

In this section we will see the various studies and research conducted in order to identify the current scenarios and trends in digital learning and also the attempts of introducing mobile devices in education.

\subsection{Main Contributions}

Kuang-Yow Lian et al. proposes a system which monitors the environmental parameters like temperatures, humidity, quality of air and the electric load. The system is implemented using smart phones. The developed system will also be able to measure the vibrations of operating machinery. For intelligent monitoring, ZigBee and Wi-Fi protocols are used. The integrated system is fabricated by 32 
bit ARM core Arduino Duo module. The measurement results were displayed using the Android and web based system.TCP/IP protocol has been used to transmit the data to a cloud device. LimitationZigbee, Wi-Fi and ARM based factory monitoring system is discussed, but the issue of cost and code size has not been tackled in this paper. It is not a cost effective solution. V. Vanitha et al. discusses an extended service oriented architecture for designing customizable sensor network and also discusses the solution to overcome the limitation of sensing systems like robustness, complexity etc. The system is basically improvement in service oriented architecture of embedded systems. Limitation - Paper describes the architecture for wireless sensor networks but doesnt discuss about application of WSN in related fields like precision agriculture etc. Dr. S. S. Riaz Ahamed in this paper the IEEE ZigBee slandered has been discussed. The paper suggests that network security is provided by Zigbee which can be implemented for small power consumption and minor cost. Limitation -Paper is all about the features of ZigBee technology, but its applications in wireless sensor networks are not included.

\section{Implementation}

\subsection{System Architecture}

Building a wireless sensor network system requires development and integration of many hardware and software components. shows the overall network structure of an monitoring wireless sensor network system that we have developed. Each node consists of an Arduino [3], an nRF24L01(+) module and a sensor. The nodes are identified by their number $(00,01,011$ etc.) depending on their position in the network. The node 00 is the coordinator node. This is Base node to which all the other nodes in the network send their information to. Each node in the network can be powered up using a battery. However, it is not advised for the coordinator or the super nodes [6] to be powered by battery. The radio communication between the nodes is shown below in figure.

\subsection{Working}

Temperature sensors are deployed at various locations in the industry. Each Sensors is connected with Arduino board and NRF24L01 which is highly integrated, ultra low power (ULP) 2Mbps RF transceiver IC. Sensors sends the sensed data to the router. which sends it to the Gateway Node. In WSN, Each Node is connected to sub-coordinator nodes or routers and each sub-coordinator node is connected to coordinator node or gateway node. The gateway node collects all the data from the sensors and sends it to the cloud for further processing here servlets are used to process the data. A threshold is Set by the User and if the temperature gets out of the threshold then the system alerts the administrator via SMS.

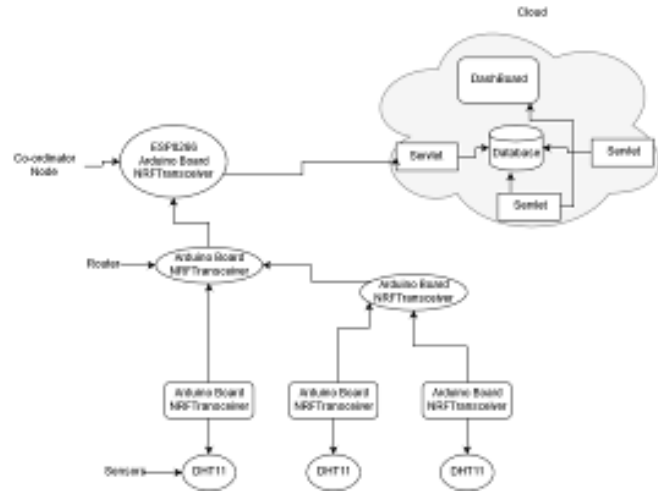

\section{Hardware and Software Requirement 4.1. Hardware Requirement 4.1.1. Arduino Nano Board}

The Arduino Nano is a little, complete, and breadboardaccommodating board dependent on the ATmega328 (Arduino Nano 3.x) or ATmega168. It has pretty much a similar usefulness of the Arduino Duemilanove, however in a different bundle. Arduino Nano V3.0 It needs just a DC control jack, and works with a Mini-B USB link rather than a standard one.The Arduino Nano can be controlled by means of the Mini-B USB association, 6-20V unregulated outer power supply (stick 30 ), or $5 \mathrm{~V}$ directed outside power supply (stick 27). The power source is naturally chosen to the most noteworthy voltage source. is the Arduino Nano gadget.

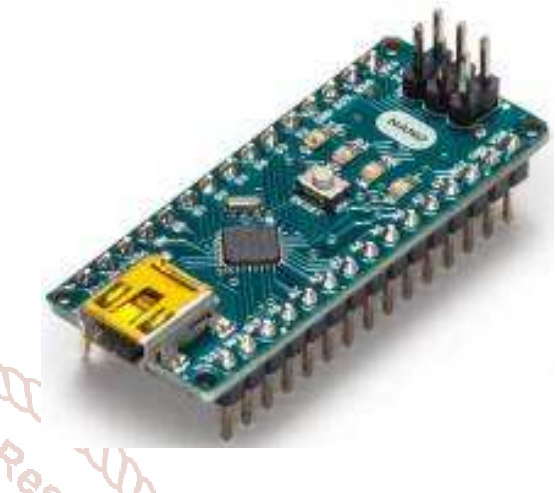

4.1.2. ESP8266

The ESP8266 is a minimal effort Wi-Fi microchip with full TCP/IP stack and microcontroller ability created by Shanghai-based Chinese producer Espressif Systems. The chip first went to the consideration of western producers in August 2014 with the ESP01 module, made by an outsider maker Ai-Thinker. This little module enables microcontrollers to interface with a Wi-Fi system and make straightforward TCP/IP associations utilizing Hayes-style directions. Be that as it may, at the time there was no English-language documentation on the chip and the directions it acknowledged. The exceptionally low cost and the way that there were not many outer parts on the module, which recommended that it could in the long run be extremely economical in volume, pulled in numerous programmers to investigate the module, chip, and the product on it, just as to decipher the Chinese documentation

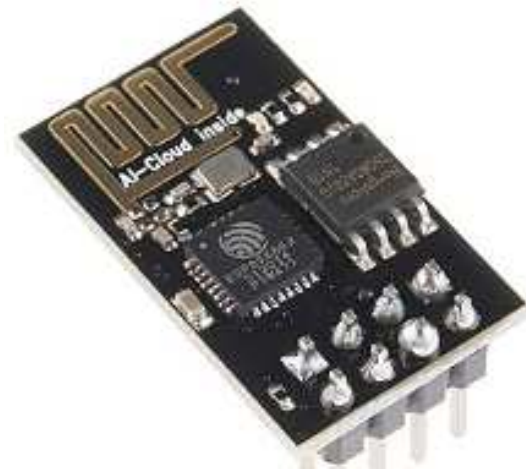

\subsubsection{GSM Board}

The Arduino GSM Shield 2 permits an Arduino board to associate with the web, make/get voice calls and send/get SMS messages. The shield utilizes a radio modem M10 by Quectel. It is conceivable to speak with the board utilizing AT directions. The GSM library has countless for correspondence with the shield. The shield utilizes advanced pins 2 and 3 for programming sequential correspondence 
with the M10. Stick 2 is associated with the M10s TX stick and stick 3 to its RX stick. See these notes for working with an Arduino Mega, Mega ADK, or Leonardo. The modem's PWRKEY stick is associated with Arduino stick 7. The M10 is a Quad-band GSM/GPRS modem that works at frequencies GSM850MHz, GSM900MHz, DCS1800MHz and PCS1900MHz. It underpins TCP/UDP and HTTP conventions through a GPRS association. GPRS information downlink and uplink exchange speed most extreme is $85.6 \mathrm{kbps}$. To interface with the cell arrange, the board requires a SIM card given by a system administrator.

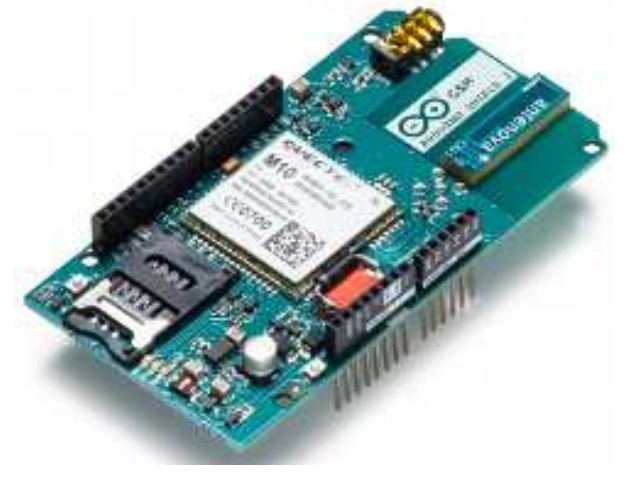

\subsection{4 nRF24L01}

nRF24L01 is a solitary chip radio handset for the worldwide $2.4-2.5 \mathrm{GHz}$ ISM band. The handset comprises of a completely incorporated recurrence synthesizer, a power amplifier, a precious stone oscillator, a demodulator, modulator and Enhanced ShockBurst convention motor. Yield control, recurrence channels, and convention setup are effectively programmable through a SPI interface. Current utilization is exceptionally low, just $9.0 \mathrm{~mA}$ at a yield intensity of $-6 \mathrm{dBm}$ and $12.3 \mathrm{~mA}$ in RX mode. Worked in Power Down and Standby modes makes control sparing effectively feasible.

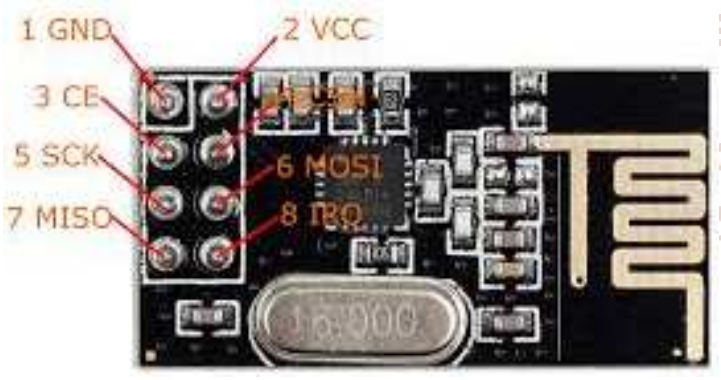

\subsubsection{DHT11 Sensor}

The DHT11 is a fundamental, ultra ease advanced temperature and mugginess sensor. It utilizes a capacitive dampness sensor and a thermistor to gauge the encompassing air, and releases a computerized flag on the information stick (no simple info pins required). Its genuinely easy to utilize, however requires cautious planning to get information. The main genuine drawback of this sensor is you can just get new information from it once at regular intervals, so when utilizing our library, sensor readings can be as long as 2 seconds old.

$>$ Low expense

$>3$ to $5 \mathrm{~V}$ power and $\mathrm{I} / \mathrm{O}$

$>2.5 \mathrm{~mA}$ max current use amid change (while mentioning information)

$>$ Good for 20-80

$>$ Good for $0-50 \mathrm{C}$ temperature readings $2 \mathrm{C}$ precision

$>$ No more than $1 \mathrm{~Hz}$ examining rate (when consistently)

$>$ Body estimate $15.5 \mathrm{~mm} \times 12 \mathrm{~mm} \times 5.5 \mathrm{~mm}$

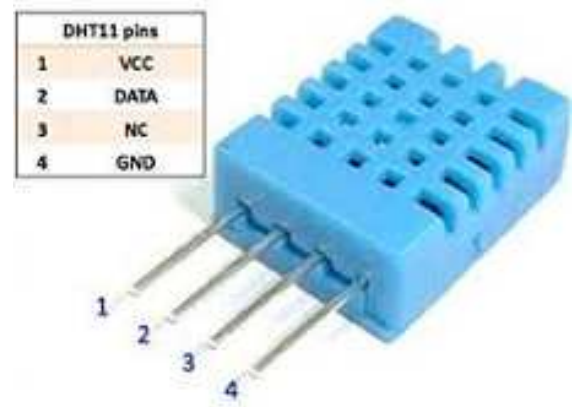

\subsection{Software Requirement \\ 4.2.1 Arduino IDE}

The Arduino coordinated advancement condition (IDE) is a cross-stage application (for Windows, macOS, Linux) that is written in the programming language Java. It is utilized to compose and transfer projects to Arduino board. The source code for the IDE is discharged under the GNU General Public License, variant 2. The Arduino IDE bolsters the dialects C and $\mathrm{C}++$ utilizing uncommon guidelines of code organizing. The Arduino IDE supplies a product library from the Wiring venture, which gives numerous basic information and yield strategies. Client composed code just requires two fundamental capacities, for beginning the sketch and the primary program circle, that are gathered and connected with a program stub principle(0 into an executable cyclic official program with the GNU toolchain, likewise included with the IDE dissemination. The Arduino IDE utilizes the program avrdude to change over the executable code into a content file in hexadecimal encoding that is stacked into the Arduino board by a loader program in the board's firmware.

\section{Modeling}

\subsection{Use Case Diagram}

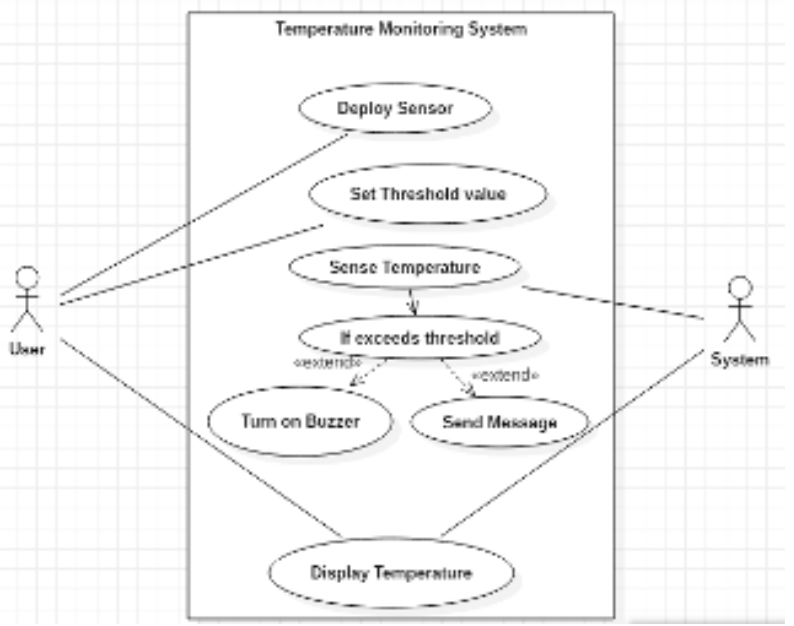

\subsection{Class Diagram}

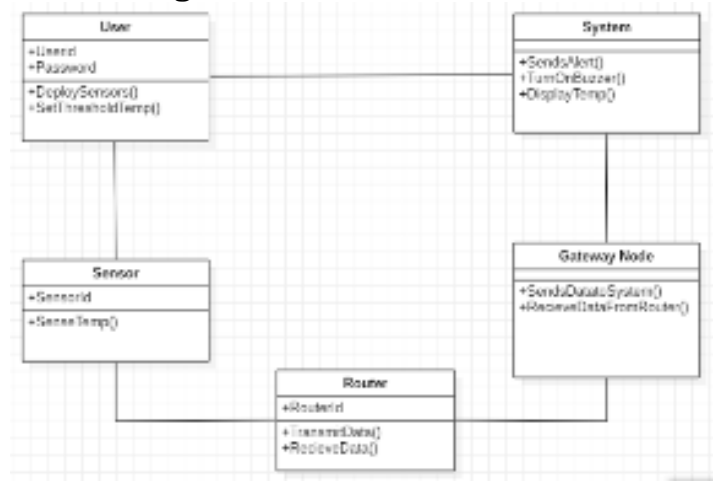




\subsection{Activity Diagram}

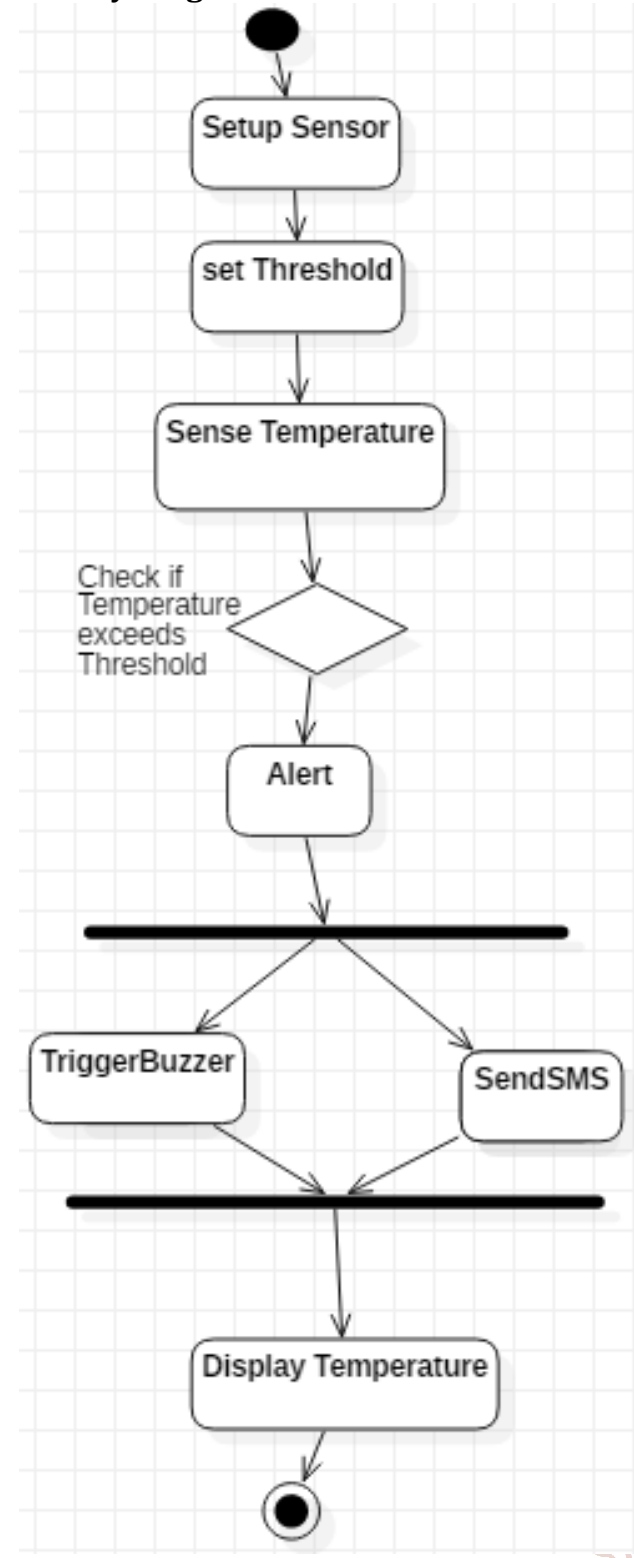

6. Advantages and Disadvantages

\subsection{Advantages}

$>$ No Wiring Constraints: Wireless sensor hubs are introduced on modern gadgets and hardware's to screen the temperature. Without the wiring requirements, gadgets can be used in applications that beforehand are either physically inaccessible or cost restrictive.

$>$ Easy Maintenance: Wireless gadget is nearly lighthearted, just a battery change is essential following quite a while of activity. Moreover, it is likewise conceivable to move current remote gadgets or send extra remote gadgets on the control framework after it has been introduced with negligible changes to the current configuration.
Detter Performance: Industrial WSNs can possibly beat the current procedure control coordinate with wired gadgets. Right off the bat, it has higher information transmission speed.

\subsection{Disadvantages}

WSN systems are not verify when contrasted with wired systems. Programmers can without much of a stretch hack the system.

$>$ Nodes should be charged at ordinary interims. Battery life of the hubs is exceptionally low.

$>$ Communication speed is nearly low than the wired system.

$>$ Wireless sensor systems continue diverting by different remote gadgets.

\section{Conclusion}

In this paper, the design method for a temperature monitoring application using a wireless sensor network (WSN) is proposed. This report has successfully demonstrated the application of the WSN to monitor the industry temperature.

\section{Bibliography}

[1] A low-cost, compact indoor thermal environment monitor;Song-Chen, K. Watanabe, M. Nakayama, M. Kanehori, H. Okuyama, Y. Ito, and E. Kushime.

[2] Simultaneous Measurement of Humidity and Temperature by Combining a Reflective Intensity-Based Optical Fiber Sensor and a Fiber Bragg Grating ;Francisco J. Arregui, Member, IEEE, Ignacio R. Matas, Member, IEEE, Kristie L. Cooper, and Richard O. Claus, Senior Member, IEEE.

[3] Distributed Remote Temperature Monitoring and Acquisition System Based on CAN Bus ;Zhu Qishen,Zhu Dongmei, Su Xunwen, Nanjing Institute ofIndustry Technology, Nanjing Jiangsu, 210046,China.

[4] Research on the Temperature Humidity Monitoring System in the Key Areas of the Hospital Based on the Internet of Things ;Benzhen Guo1,Xiaojing Wang, Xiao Zhang,Jingjing Yang1,Zhihui Wang1 1School of Information Science and Engineering, Hebei North University, Zhangjiakou075000, Hebei, China,Department of Humanities and Social Sciences, Zhangjiakou University, Zhangjiakou075000, Hebei, China.

[5] ZIGBEE BASED SENSOR NETWORKS FOR TEMPERATURE MONITORING AND CONTROLLING;Sachin S. Patil1,Shrenik S. Sarade2,Sagar V. Chavan3 1, 2(Department of Electrical Engineering, ADCET ASHTA/Shivaji University, Kolhapur, India) 3(Department of Electrical Engineering, RIT Sakharale/Shivaji University, Kolhapur, India. 